\title{
TONSILLECTOMY IN IgA NEPHROPATHY - CLINICAL CASE
}

\author{
LIVIA MIRELA POPA ${ }^{1}$, LIVIA POPA ${ }^{2}$, CĂTĂLIN-BOGDAN OSALCIUC ${ }^{3}$ \\ 1 “Lucian Blaga” University of Sibiu. 2,3 "Sibiu County Clinical Emergency Hospital
}

Keywords: nephropathy, tonsillectomy, macroscopic hematuria

IgA Abstract: This article presents the clinical case of IgA nephropathy of a patient hospitalized in order to investigate a nephritic syndrome, apparently with acute onset, discovered in the following circumstances: recurrent macroscopic hematuria, decreased urinary volume, the first episode occurring about a month ago in the context of an acute infection of upper airways, remitted under antibiotic therapy, with reappearance every 2 weeks, also accompanied by odynophagia and decreased urinary volume.

\section{INTRODUCTION}

$\operatorname{Ig} \mathrm{A}$ nephropathy was described by Berger and Hinglais in 1968 .

IgA nephropathy is a form of chronic glomerulonephritis, defined by $\operatorname{IgA}$ immune complexes deposits in the glomeruli. The most common manifestations are persistent or recurrent macroscopic hematuria (in $90 \%$ of affected children) or asymptomatic microscopic hematuria with mild proteinuria.(1) Massive hematuria from $\operatorname{IgA}$ nephropathy usually begins 1-2 days after a febrile disease at mucosa level (upper respiratory tract, sinus, enteric), thus mimicking acute glomerulonephritis, except that the onset of hematuria is earlier, coinciding with the febrile disease or appearing immediately after it, accompanied by lumbar pain. $(1,2)$

As clinical features, in addition to recurrent episodes of macroscopic hematuria and persistent microscopic hematuria, there is also the nephritic syndrome which is rare (5\%) and corresponds to a histological form with minimal lesions, acute renal failure (5\%) associated with either an episode of macroscopic hematuria (acute tubular necrosis), or with extracapillary proliferation, chronic renal failure (10-20\%) and hypertension that can sometimes take a malignant form.(3)

From a pathogenetic point of view, IgA nephropathy is a nephropathy with immune complexes. The following fundamental abnormalities are involved in its pathogenesis: increased production of Ig $\mathrm{A}$ in the mucosal immune system (MALT) and bone marrow; abnormal glycosylation of serum IgA 1 molecules; intense storage of abnormally glycosylated polymeric $\operatorname{IgA} 1$ in the glomerular mesangium; glomerular inflammatory process, induced by $\operatorname{IgA}$ deposits. $(1,4)$

$\operatorname{IgA}$ nephropathy is the most common glomerular nephropathy worldwide, the reported incidence of the disease varies in the world between 0.2 and $2.8 \% .000$ / year. However, the real incidence is, most probably, much underestimated, because most patients are asymptomatic, being detected only by systematic urine examinations.(4)

It is unequally distributed geographically: USA 5\% total cases, $10 \%$ glomerulopathies; Europe: $15 \%$ total cases,
20\% glomerulopathies; Asia: $30 \%$ total cases, $40 \%$ glomerulopathies; Japan: $50 \%$ glomerulopathy. IgA nephropathy mainly affects the male sex (male/female ratio is $2 / 1$ ), and can occur at any age, but the maximum incidence is between 20 and 40 years old, being rare over the age of 50.(5)

\section{CASE PRESENTATION}

A 42-year-old patient was admitted for the investigation of an apparently acute nephritic syndrome discovered in the following circumstances: recurrent macroscopic hematuria, the first episode appeared about a month before, in the context of an acute upper airway infection, remitted under antibiotic therapy, with recurrence within 2 weeks, also accompanied by odynophagia and decreased urinary volume.

From the significant personal medical history, we mention: arterial hypertension in treatment with ACE inhibitors, Moldamin treatment for a period of 6 months 10 years before, for repeated streptococcal angina, urinary syndrome mentioned in medical documents one year ago ( 250 red blood cells, 75 $\mathrm{mg} / \mathrm{dl}$ protein, normal renal function), and one month before admission-active urinary sediment (hematuria, proteinuria) and minor nitrogen retention syndrome (creatinine $1.61 \mathrm{mg} / \mathrm{dl}$ ).

The objective clinical examination at admission revealed the following: good general condition, afebrile, the temperature being measured every 4 hours, mild odynophagia, congested tonsils and discrete pharyngeal erythema, no edema, no changes in pulmonary stetacoustics, increased blood pressure $160 / 100 \mathrm{mmHg}$ in both arms, both in supine position and in orthostatism, spontaneous diuresis over $2000 \mathrm{ml} / 24 \mathrm{~h}$, without hematuria at the time of hospitalization. On the occasion of this hospitalization there is an aggravation of nitrogen retention (serum creatinine $4.11 \mathrm{mg} / \mathrm{dl}$ ) and persistence of nephritic syndrome (urinary sediment with frequent dysmorphic red blood cells and granular cylinders, subnephrotic proteinuria $-2.3 \mathrm{~g} /$ 24h).

Presumptive diagnosis: Acute renal failure. Acute nephritic syndrome. Recurrent macroscopic hematuria. Chronic tonsillitis. Hypertension.

${ }^{1}$ Corresponding author: Livia Mirela Popa, B-dul. C. Coposu, Nr. 3-5, Sibiu, România, E-mail: liviamirelapopa@ yahoo.com, Phone: +40269215050 Article received on 18.06.2021 and accepted for publication on 27.08.2021 


\section{CLINICAL ASPECTS}

Abdominal ultrasound performed upon hospitalization revealed: homogeneous sonic liver, hyperechoic, with posterior acoustic attenuation, of normal size; shielded pancreas due to gas overlaps; gallbladder with thin walls, transonic content; right kidney with a long axis of about $12.5 \mathrm{~cm}$, cortical index $24 \mathrm{~mm}$, cortico-medullary differentiation present, without dilatations of the excretory tract, intrarenal vascularization visible up to the level of arched arteries; spleen with dimensions of 10/3.5 cm, homogeneous echostructure; left kidney with $11.8 \mathrm{~cm}$ long axis, cortical index $25 \mathrm{~mm}$, vascularization similar to that of the right kidney; bladder in semi-vacuum.

Investigations with etiological aim were started, performing infectious, immunological and neoplastic screening:

pulmonary radiography without pleuro-pulmonary evolutionary lesions;

pharyngeal exudate, nasal exudate, urine culture without bacterial growth;

there are no clinical signs of subacute endocarditis;

HBs negative, HIV negative; Ac HCV negative;

no VDRL/TPHA dissociation is found;

the available immunology is all negative (ASLO, C3, C4, ANA profile, Ig G, M, pANCA, cANCA) except for the increased titer of Ig A (653U / L), cryoglobulins absent; neoplastic markers are negative (PSA, CEA, serum alpha fetoprotein); there is a slightly increased serum level of the CA19.9 marker that cannot be framed in a clinical context. further investigations by contrast-enhanced abdominal computed tomography were considered, an investigation required in the context of significant nitrogen retention.

The patient has been known for about 1 year with type 2 diabetes, diet-controlled. The glycemic values at repeated determinations were kept below $150 \mathrm{mg} / \mathrm{dl}$, not requiring the administration of hypoglycemic medication: glycosylated hemoglobin was $6.4 \%$. He also mentioned the presence of a lumbar discopathy for which he underwent physical treatment (physiotherapy, massage) in February this year, prior to the onset of hematuria. In this context, a monoclonal gammopathy was discussed - diagnosis unsupported by available laboratory tests (normal serum calcium, normal serum proteins, absent Bence Jones proteins, serum protein electrophoresis: albumin 46.1\%, Alpha 1-globulin 4\%, Alpha 2- globulins 14.2\%, Betaglobulins $18.4 \mathrm{~g} / \mathrm{L}$, Beta 1-globulins 9.3\%, Beta 2-globulins 9.1\%, Gamma-globulins $17.1 \%$, albumin/globulin ratio 0.86 )

The ENT consultation confirmed our diagnosis of chronic catarrhal tonsillitis and revealed a left submandibular inflammatory lymphadenopathy.

The suspicion of a poststreptococcal glomerulonephritis was raised, a diagnosis ruled out by the negative pharyngeal exudate and the normal ASLO titer.

Under water intake of about $2000-2500 \mathrm{ml} / 24 \mathrm{~h}$, alkalizing medication, 7-day antibiotherapy (Cefuroxime), antifungal and probiotic protection, pharyngeal disinfectants and hypotensive medication, the evolution was favourable, with remission of odynophagia, spontaneous diuresis of about 2500 $\mathrm{ml} / 24$, nitrogen retention with a slow degree of reversibility (3.4mg/dl serum creatinine on 22.03.2020).

The case was interpreted as an acute renal failure with rapid progressive evolution (doubling of creatinine levels every 4 weeks) most likely against the background of $\operatorname{IgA}$ nephropathy, reason for which the patient was guided to perform a renal biopsy with the following histopathological result: glomeruli which showed slight mesangial expansion; some had fibrous crescents; tubules showed moderate, focal atrophy; at the level of the interstitium present, reduced lymphocytic inflammatory infiltrate; trichrome staining highlighted interstitial fibrosis; IgG negative; intensely positive $\mathrm{IgA}$; rare IgM positive hyaline cylinders; $\mathrm{C} 3$ and fibrinogen negative; the conclusion being-> the morphological changes described may belong to IgA nephropathy with a METS score $1+1+0+0$. nephropathy.

The diagnosis established at discharge was: $\operatorname{IgA}$

Figure no. 1. Electron microscopy - mesangial electrondense deposits

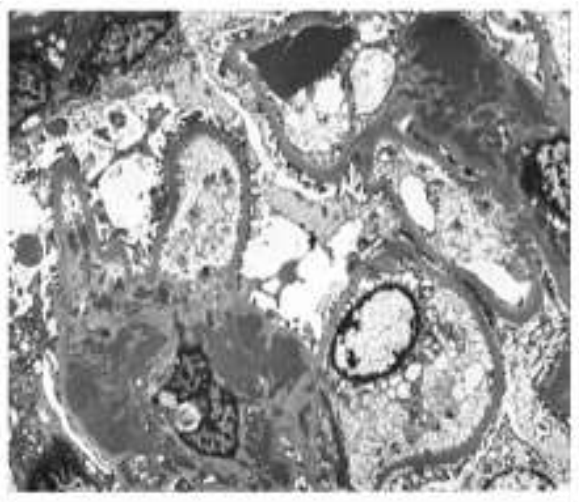

Figure no. 2. Optic microscopy

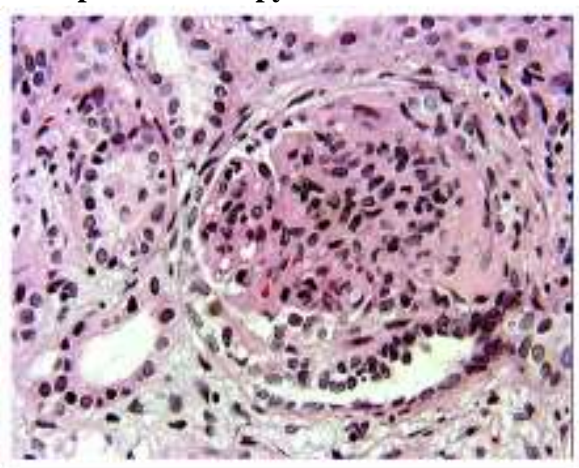

DISCUSSIONS

Considering the clear evidence of the degradation of renal function at each outbreak of acute tonsillitis, as a treatment alternative, the decision was to perform tonsillectomy.

One study evaluated the eefficacy of tonsillectomy in the long-term outcome of 118 patients. $(6,7)$

From the point of view of the therapeutic approach, the decision to perform a tonsillectomy was an effective one. After performing the intervention, at a distance of approximately one year, the patient did not have exacerbations and the nitrogen retention syndrome remained constant at reasonable values.

For tonsillectomy, as a treatment option in $\operatorname{IgA}$ nephropathy, we do not yet have enough definite data. This has given favorable results in some retrospective, unrandomized studies, mostly conducted in Japan. Likewise, a retrospective 10-year follow of 71 patients and a third retrospective trial of 200 Japanese patients found improved survival with tonsillectomy. $(3,8)$ It is a popular therapeutic option in this country, especially in combination with corticosteroid therapy.(5)

A meta-analysis of seven studies studies including 858 patients found more remissions and less end-stage renal disease (ESRD) in the tonsillectomized patients, but only in those who received corticosteriods as well.(7)

Another option would be polyunsaturated omega-3 fatty acids due to their anti-inflammatory, antihypertensive and lipid-lowering properties. Kidney transplantation: IgA deposits appear on the grafted kidney in $2 / 3$ of cases in the first 2-3 months; the evolution of the graft is good in general (10 years). $(4,5)$ 


\section{CLINICAL ASPECTS}

The prognosis depends on several unfavorable factors such as: proteinuria $>0.5 \mathrm{~g} / \mathrm{day}$; high blood pressure; persistent microscopic hematuria; MEST-S histological score; old age; chronic advanced kidney disease.

The evolution is prolonged and often unpredictable, complete spontaneous remissions are possible in 5-30\% of cases more common in children and very rare in adults. Recurrent episodes of macroscopic hematuria, secondary to infections, are relatively common, some accompanied by irreversible acute renal failure. $(4,5)$

\section{CONCLUSIONS}

The peculiarity of the case consists in the favorable evolution after performing the tonsillectomy, a fact that could bring into discussion routinely performing tonsillectomy in patients diagnosed with IgA Nephropathy

\section{REFERENCES}

1. Appel GB. IgA Nephropathy: Clinical Features, Pathogenesis, and treatment. In H. Trachtman et al (eds.), Glomerulonephritis, Springer Nature Switzerland AG; 2019.

2. Beers MH, Robert S. Porter RS. Manualul Merck de diagnostic si tratament. Editia A XVIII-A, Editura ALL; 2009.

3. Agaki T, Kosaka M, Hattori K, et al. Long-term results of tonsillectomy as a treatment for IgA nephropathy. Acta Otolaryngol Suppl. 2004;555:38.

4. Covic A. Nefrologie. Principii teoretice si practice. Casa editoriala Demiurg. Iasi; 2011.

5. Ungureanu G, Covic A. Terapeutica medicală, Editura Polirom; 2014.

6. Wang Y, Chen J, et al. A meta-analysis of the clinical remission rate and long-term efficacy of tonsillectomy in patients with IgA nephropathy. Nephrol Dial Transplant. 2011;26:1923.

7. Xie $Y$, Chen $X$, Nishis $S$, et al. The efficacy of tonsillectomy on long-term renal survival in patients with IgA nephropaty, Kidney Int. 2003;63:1861.

8. Maeda I, Hayashi T, Sato KK, et al. Tonsillectomy has beneficial effects on remission and progression of $\operatorname{IgA}$ nephropaty independent of steroid therapy. Nephrol Dial Transplant. 2012;27:2806-2813. 